\title{
Lighting Simulation in 3D Printing (Abstract)
}

\author{
Denis Sumin ${ }^{1}$, Karol Myszkowski ${ }^{1}$, \\ Alexey Voloboy ${ }^{2}$ \\ ${ }^{1}$ Max-Planck-Institute for Informatics, \\ Saarbrucken, Germany \\ ${ }^{2}$ The Keldysh Institute of Applied Mathematics RAS, Moscow, Russia \\ da.suminegmail.com
}

3D printing for rapid prototyping and production of unique objects is being actively developed. Consumer-grade printers are now commonly available for a range of purposes, while increasingly advanced techniques allow us to fabricate novel shapes, mechanical properties, and appearances. The printers' capabilities have improved dramatically from printing single-material objects to producing detailed structures with per-voxel material variation. Since the 2010s, it is possible to fabricate fullcolour 3D objects with resolutions of hundreds of DPI (voxels' dimensions are in the order of $10 \mu \mathrm{m}$ ). Such capabilities are most prominent in printers based on the photopolymer jetting process. Ideally, it should be possible now to produce photorealistic appearances or visually indistinguishable objects copies for, e.g., cultural-heritage applications.

However, the resins used as print materials in commercial devices are inherently translucent, i.e., exhibit significant sub-surface scattering. This serves effective colour mixing in full-colour print processes, thus commercial printer drivers offer highquality colour reproduction. At the same time, the resulting light diffusion leads to over-blurring and potential colour bleeding when printing spatially-varying colour textures. This translucent 'crosstalk' between surface points also strongly depends on the internal structure of the volume surrounding each surface point.

Previously existing scattering-aware methods used simplified models for light diffusion and accepted the visual blur as an immutable property of the print medium.

In this talk, we present the series of works conducted by a consortium of several institutes (Max-Planck Institute for Informatics, Germany; Charles University in Prague, Czech Republic; Institute of Science and Technology, Austria; University College London, United Kingdom; Universita della Svizzera Italiana, Switzerland; The Keldysh Institute of Applied Mathematics RAS, Russia). Our work counteracts heterogeneous scattering to obtain the impression of a crisp albedo texture on top of the 3D print, by optimizing for a fully volumetric material distribution that preserves the target appearance.

We build our iterative method on top of a general Monte-Carlo simulation of heterogeneous scattering. We find out that a certain arrangement of materials expands the gamut of achievable appearances and makes it possible to produce sharp textures.

Copyright (C) 2020 for this paper by its authors. Use permitted under Creative Commons License Attribution 4.0 International (CC BY 4.0). 


\section{D. Sumin et al.}

This knowledge built-in into the volume-update step enables convergence just after $10-15$ iterations. We verify these findings using an established stochastic gradientdescent optimization for small canonical objects where it is feasible computationally.

Expansion of our method to fabrication of arbitrary 3D objects with the translucent resins opens a set of problems of achievable colour combinations on the two sides of thin shapes, in the extreme convex and concave shapes. Physically correct lighting simulation enables exploration of these extreme cases where no ideal solution is possible. It turns out that a re-formulation of established gamut-mapping methods is needed for the medium with the inherent cross-talk properties such as the scattering resins of the modern full-colour 3D printers.

Elaborating further ideas from we also propose a fast forward predictor of the object's surface appearance based on a neural network to replace the Monte-Carlo simulation in order to speed up the preparation of the model by 300 times. The achieved acceleration allows to reduce simulation time to minutes for a single, GPU-equipped workstation. This makes the print preparation timings practical. 